\title{
Linkage analysis of left ventricular outflow tract malformations (aortic valve stenosis, coarctation of the aorta, and hypoplastic left heart syndrome)
}

\author{
Kim L McBride ${ }^{*, 1,2}$, Gloria A Zender ${ }^{1}$, Sara M Fitzgerald-Butt ${ }^{1}$, Daniel Koehler ${ }^{1}$, \\ Andres Menesses-Diaz ${ }^{3}$, Susan Fernbach ${ }^{3}$, Kwanghyuk Lee ${ }^{3}$, Jeffrey A Towbin ${ }^{4}$, \\ Suzanne Leal ${ }^{3}$ and John W Belmont ${ }^{3}$
}

\footnotetext{
${ }^{1}$ Center for Molecular and Human Genetics, The Research Institute at Nationwide Children's Hospital, Columbus, OH, USA; ${ }^{2}$ Department of Pediatrics, College of Medicine, Ohio State University, Columbus, OH, USA; ${ }^{3}$ Department of Molecular and Human Genetics, Baylor College of Medicine, Houston, TX, USA and ${ }^{4}$ Department of Pediatrics, Section of Cardiology, Baylor College of Medicine, Houston, TX, USA
}

The left ventricular outflow tract (LVOT) malformations aortic valve stenosis (AVS), coarctation of the aorta (CoA), and hypoplastic left heart syndrome (HLHS) are significant causes of infant mortality. These three malformations are thought to share developmental pathogenetic mechanisms. A strong genetic component has been demonstrated earlier, but the underlying genetic etiologies are unknown. Our objective was to identify genetic susceptibility loci for the broad phenotype of LVOT malformations. We genotyped 411 microsatellites spaced at an average of $10 \mathrm{cM}$ in 43 families constituting 289 individuals, with an additional $5 \mathrm{cM}$ spaced markers for fine mapping. A non-parametric linkage (NPL) analysis of the combined LVOT malformations gave three suggestive linkage peaks on chromosomes 16p12 (NPL score $(N P L S)=2.52), 2 p 23(N P L S=2.41)$, and 10q21 (NPLS=2.14). Individually, suggestive peaks for AVS families occurred on chromosomes $16 \mathrm{p} 12(\mathrm{NPLS}=2.64), 7 \mathrm{q} 36(\mathrm{NPLS}=2.31)$, and 2p25 (NPLS = 2.14); and for CoA families on chromosome 1q24 (NPLS=2.61), 6p23 (NPLS=2.29), 7p14 (NPLS=2.27), $10 q 11$ $(N P L S=1.98)$, and $2 p 15(N P L S=2.02)$. Significant NPLS in HLHS families were noted for chromosome $2 p 15(N P L S=3.23)$, with additional suggestive peaks on $19 q 13(N P L S=2.16)$ and 10q21 $($ NPLS $=2.07)$. Overlapping linkage signals on 10q11 (AVS and CoA) and 16p12 (AVS, CoA, and HLHS) led to higher NPL scores when all malformations were analyzed together. In conclusion, we report suggestive evidence for linkage to chromosomes 2p23, 10q21, and 16p12 for the LVOT malformations of AVS, CoA, and HLHS individually and in a combined analysis, with a significant peak on 2p15 for HLHS. Overlapping linkage peaks provide evidence for a common genetic etiology.

European Journal of Human Genetics (2009) 17, 811-819; doi:10.1038/ejhg.2008.255; published online 14 January 2009

Keywords: heart defects; congenital; embryonic and fetal development; hereditary disease; pediatrics

*Correspondence: Dr KL McBride, Center for Molecular and Human Genetics, The Research Institute at Nationwide Children's Hospital, 700 Children's Dr, Columbus, OH 43205, USA. Tel: + 1614722 5484;

Fax: + 1614722 2817; E-mail: Kim.McBride@NationwideChildrens.org Received 28 August 2008; revised 14 October 2008; accepted 26 November 2008; published online 14 January 2009
Introduction

Congenital cardiovascular malformations (CVMs) are among the most common birth defects in the United States. At a birth prevalence of 5-8/1000 live births, they account for $\frac{1}{4}$ of all birth defects. ${ }^{1}$ Despite recent medical and surgical advances, CVMs still cause significant mortality and morbidity. 
CVMs may be classified by the specific anatomic defect, the clinical presentation (eg cyanotic $v s$ acyanotic), or the developmental mechanism perturbed during cardiogenesis. Aortic valve stenosis (AVS), coarctation of the aorta (CoA), and hypoplastic left heart syndrome (HLHS) comprise the group of CVMs that collectively result in obstruction of the left ventricular outflow tract (LVOT). These three malformations combined have an estimated birth prevalence of $1 / 1000$ and cause $15-20 \%$ of medically significant CVMs. ${ }^{1-3}$ The rates of LVOT malformations are higher in males and more common among those of White compared with those of Black ethnicity. They are also responsible for a major portion of the mortality from CVMs, in particular due to the high mortality of HLHS (1-year survival of $60-70 \%$ ) even with surgical palliation. ${ }^{4}$

The etiologies of LVOT malformations are likely complex, with environmental exposures, chromosomal abnormalities, and one or more gene defects all contributing. Exposure of the developing fetus to organic solvents or high levels of phenylalanine (as part of maternal phenylketonuria) has been associated with LVOT malformations (reviewed in Jenkins et al. ${ }^{5}$ ). These malformations may be seen in conjunction with chromosomal defects, such as $45, X$ (Turner's syndrome) ${ }^{6}$ and del11q23 (Jacobsen's syndrome). ${ }^{7}$ Overall, roughly $10-15 \%$ of individuals with an LVOT malformation have a chromosome abnormality. ${ }^{8}$ NOTCH1 mutations have been reported in two families with bicuspid aortic valve (BAV) and calcific AVS, ${ }^{9}$ in sporadic $\mathrm{BAV},{ }^{10,11}$ and recently by our group in AVS, CoA, and HLHS. ${ }^{12}$ NKX2-5 mutations have also been observed rarely in cases of LVOT malformations. ${ }^{13,14}$ However, the exact cause for most LVOT malformations is unknown.

Recurrence of these malformations in families has been reported for AVS, CoA, and HLHS. Epidemiology studies have documented a high concordance for LVOT malformations among families with multiple CVMs, although not necessarily of the same LVOT malformation within the family. Our group and others ${ }^{15,16}$ have also noted a much higher rate ( $\sim 5.5$-fold) of BAV among first-degree relatives of children with severe LVOT malformations compared with the general population, suggesting that BAV may be a forme fruste of the more serious LVOT malformations.

We went on to study the inheritance of these malformations in more detail, and showed a strong genetic component for the LVOT malformations. ${ }^{17}$ The heritability of BAV among first-degree relatives of individuals with AVS, CoA, or HLHS was estimated at 50\%, with sibling recurrence rates for AVS, CoA, or HLHS > 30. Segregation analysis was most consistent with a complex genetic inheritance involving 1-6 loci. A model of an autosomal dominant inheritance pattern with reduced penetrance was also consistent in some families.

The epidemiology studies above, combined with our recent NOTCH1 molecular genetic findings in AVS, CoA, and HLHS subjects (along with earlier reports in BAV), support the concept of a unifying genetic mechanism for an LVOT malformation phenotype. The inheritance analyses also suggest that these malformations may be caused by a limited number of genes. We thus hypothesized that LVOT malformation susceptibility loci may be identified by a traditional linkage approach using multiplex families. We report here the results of the first genome-wide linkage analysis of the broad phenotype of LVOT malformations, showing suggestive linkage signals on chromosomes 2p23, 10q21, and 16p12. Additional peaks were noted for AVS, CoA, and HLHS when analyzed separately.

\section{Materials and methods Subject recruitment}

Families were recruited from two pediatric tertiary care centers with an IRB-approved protocol. Detailed information on many families has been described earlier. ${ }^{15,17}$ Probands with AVS, CoA, and HLHS were ascertained and pedigrees obtained. Those families with at least two members affected with an LVOT malformation were included in the study. Diagnosis of the CVM was confirmed by echocardiography, cardiac catheterization, or direct observation at surgery. We defined the malformations as follows: AVS - congenital obstruction of the systemic outflow tract at the level of the valve (includes trileaflet or bileaflet aortic valve); CoA - a hemodynamically significant narrowing of the thoracic aorta, usually distal to the left subclavian artery (may or may not also have hypoplasia of the aortic arch); HLHS - mitral valve atresia or stenosis and aortic valve atresia or stenosis with hypoplasia of the left ventricle and aortic arch. We included individuals if they had a BAV in addition to their primary malformation, and also included individuals with CoA and VSD or AVS. We excluded those individuals who had interrupted aortic arch or Shone complex, any other type of CVM in addition to an LVOT malformation (except as mentioned earlier), a known chromosomal abnormality, or were diagnosed with a specific syndrome. We also gathered detailed clinical data, including pregnancy, medical and surgical histories on each family member enrolled. Wherever possible, immediate family members underwent echocardiography to assess for any structural abnormalities. Any individuals identified with a BAV on echocardiography were considered to be affected. Blood samples were collected, and lymphocytes were transformed using EBV to establish cell lines. DNA was extracted from the transformed lymphoblasts with the PureGene kit (Gentra, Minneapolis, MN, USA).

\section{Genotyping}

We performed genotyping using the Applied Biosystems (Foster City, CA, USA) medium-density linkage mapping set. This set contains 411 dinucleotide microsatellite markers spaced on average at $10 \mathrm{cM}$ throughout the 
genome. After PCR as per the manufacturer's protocols, products were pooled and the fragments were resolved on an ABI 3100 or 3730 sequencer. Additional markers spaced on average at $5 \mathrm{cM}$ were selected from the high-density linkage mapping set for chromosomes 2 (6 markers), 10 (7 markers), and 16 (4 markers) for more detailed mapping around the highest linkage peaks identified in the initial genotyping.

\section{Allele calling and quality control metrics}

Allele calling and initial quality checks were carried out with the Applied Biosystems proprietary GeneMapper software. Internal replicates (a randomly selected individual) and an external control (CEPH sample 1347-02) were used for each marker for quality control. The genotyping discrepancy was $0.86 \%$. Data were inspected for genotyping errors initially with the program PEDCHECK, ${ }^{18}$ and then with the error routine contained in MERLIN ${ }^{19}$ to identify unlikely genotypes from double recombination events. Any identified inconsistencies were returned to the laboratory for re-genotyping.

\section{Data analysis}

Parametric analysis was performed with the program MERLIN using a dominant model with reduced penetrance (80\%) and no phenocopies, based on our earlier segregation analysis. ${ }^{17}$ Heterogeneity LOD (HLOD) scores were calculated in a multipoint linkage analysis, in order to account for the possibility that not all families may segregate for the LVOT malformation at the same locus. The proportion of families contributing to the linkage peak (designated as $\alpha$ ) was recorded. Non-parametric linkage analysis was executed in the ALLEGRO linkage package. ${ }^{20}$ Non-parametric linkage scores (NPLS) were calculated in a multipoint analysis using the $S_{\text {all }}$ allele-sharing score functions. Analysis was performed first for all the three malformations as a combined LVOT phenotype, and then separately by individual diagnosis. Suggestive LOD and NPL score cut points were $>1.90$, and significant scores were $>3.0$.

Power calculations were also performed for the parametric linkage analysis, using the same parameters. Pedigrees were entered into the SIMULATE program, ${ }^{21}$ and 1000 replicates were created. We ran these in SLINK ${ }^{22,23}$ to generate expected LOD scores. Assuming a penetrance of $80 \%$ and locus homogeneity, the expected LOD scores for these pedigrees are 5.66, 5.09, and 4.45 at recombination rates $(\theta)$ of $0.0,0.05$, and 0.10 , respectively. The power to detect linkage at a LOD score of $>3.0$ is 0.97 (95\% confidence interval of $0.964-0.985$ ).

\section{Sequencing}

Direct sequencing was performed with dideoxy BigDye terminator chemistry and run on a capillary sequencer (ABI3730).
Table 1 Anatomic malformations of subjects (proband and additional affected relatives) by sex

\begin{tabular}{lrrr}
\hline Malformation & Male & Female & Total \\
\hline BAV & 14 & 9 & 23 \\
AVS & 18 & 6 & 24 \\
CoA & 28 & 5 & 33 \\
HLHS & 12 & 4 & 16 \\
Other & 1 & 4 & 5 \\
Normal & 72 & 78 & 150 \\
Unknown & 26 & 12 & 38 \\
Total & 171 & 118 & 289 \\
\hline
\end{tabular}

AVS, aortic valve stenosis; BAV, bicuspid aortic valve; CoA, coarctation of the aorta; HLHS, hypoplastic left heart syndrome.

Other includes pulmonic valve stenosis, mitral valve stenosis or valve abnormality,(3) dilated aortic arch.

\section{Results}

\section{Phenotyping}

A total of 44 families comprising 299 individuals were collected. One family was subsequently excluded from analysis because of non-paternity at several points in the pedigree, leaving 43 families of 289 individuals (Supplementary Figure). The average pedigree size was 6.80 (range $3-21)$.

Echocardiography studies were carried out in 251/289 individuals (Table 1). Families were divided by a hierarchy using the most severe malformation identified within the family (ranked HLHS, CoA, and AVS from worst to least) into AVS (12 families of 90 individuals), CoA (20 families of 124 individuals), or HLHS (11 families of 75 individuals). All the AVS families had multiple individuals with aortic valve disease; 2 of 12 probands had BAV and AVS. Ten of the CoA families had two or more individuals with a CoA, some of which had additional members with AVS or BAV. The other ten CoA families had one member with a CoA and one or more members with congenital AVS or BAV. Among the HLHS families, five had two individuals with HLHS, and one had an individual with HLHS and one with CoA. Some of these HLHS families had additional affected members with AVS or BAV. The remaining of the HLHS families had one member with HLHS and other family members with congenital AVS or BAV.

\section{Genome-wide linkage scan}

The results of the linkage analysis on the initial $10 \mathrm{cM}$ genome-wide scan are shown in Figures 1 and 2, Table 2 (all LVOT phenotypes) and the Supplementary Table (AVS, CoA, and HLHS data separately). We found evidence of suggestive linkage on chromosome 2p23.2 (parametric HLOD = 2.20; NPLS $=2.25$ ), chromosome 10q21.2 (parametric HLOD = 0.89 ; NPLS $=2.57$ ), and chromosome 16p12.2 (parametric $\mathrm{HLOD}=2.25 ; \mathrm{NPLS}=2.03$ ). Analysis of each malformation individually noted overlapping peaks and additional peaks unique to each malformation (Figures 1 and 2). AVS malformation families had suggestive peaks on chromosomes 

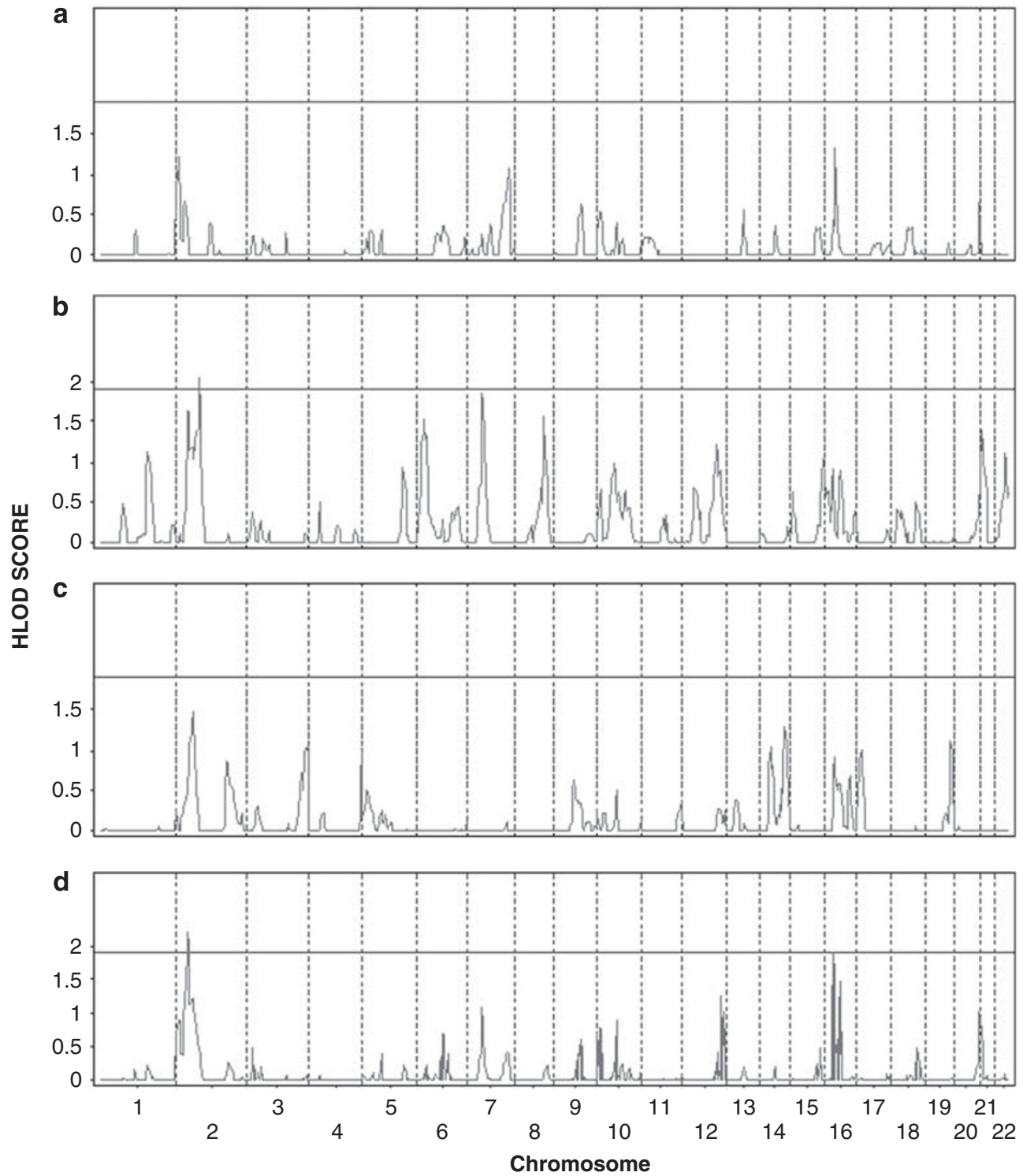

Figure 1 Genome-wide heterogeneity LOD (HLOD) score results for 43 families with left ventricular outflow tract (LVOT) malformations in the initial $10 \mathrm{cM}$ scan, by chromosome and position. HLOD score results for (a) AVS families, (b) CoA families, (c) HLHS families, and (d) the combined LVOT malformation phenotype. The line indicates the threshold for suggestive HLOD score (1.9).

$16 \mathrm{p} 12 \quad(\mathrm{NPLS}=2.64), \quad 7 \mathrm{q} 36 \quad(\mathrm{NPLS}=2.31), \quad$ and $2 \mathrm{p} 25$ $(\mathrm{NPLS}=2.14)$; CoA families on chromosome 1q24 (NPLS $=$ 2.61), 6p23 (NPLS=2.29), 7p14 (NPLS=2.27), 10q11 $(\mathrm{NPLS}=1.98)$, and 2p15 $(\mathrm{NPLS}=1.90)$. Significant NPL scores in HLHS families were noted for chromosome 2p15 $(\mathrm{NPLS}=3.17)$, with additional suggestive peaks on chromosomes 19q13 $(\mathrm{NPLS}=2.16)$ and 10q21 $(\mathrm{NPLS}=1.94)$.

On the basis of this information, additional genotyping was performed, and analysis was repeated for chromosomes 2, 10, and 16. As the NPL analysis provided the maximal score in all cases, only those results are shown. An increase in NPL scores was obtained on chromosomes 2 and 16 with the additional more closely spaced markers.

Analysis of the three chromosomes by individual diagnosis allows assessment of the contribution of each to the combined LVOT malformation peak (Figure 3). Chromosome 2 shows closely aligned peaks for AVS, CoA, and HLHS for $20-100 \mathrm{cM}$ genetic positions, but overlaps occur only on the shoulders of the peaks. Chromosome 10 shows two interesting areas: overlapping AVS and CoA peaks lead to a slightly higher combined peak (at position $15-20 \mathrm{cM})$, with a similar phenomenon at position 

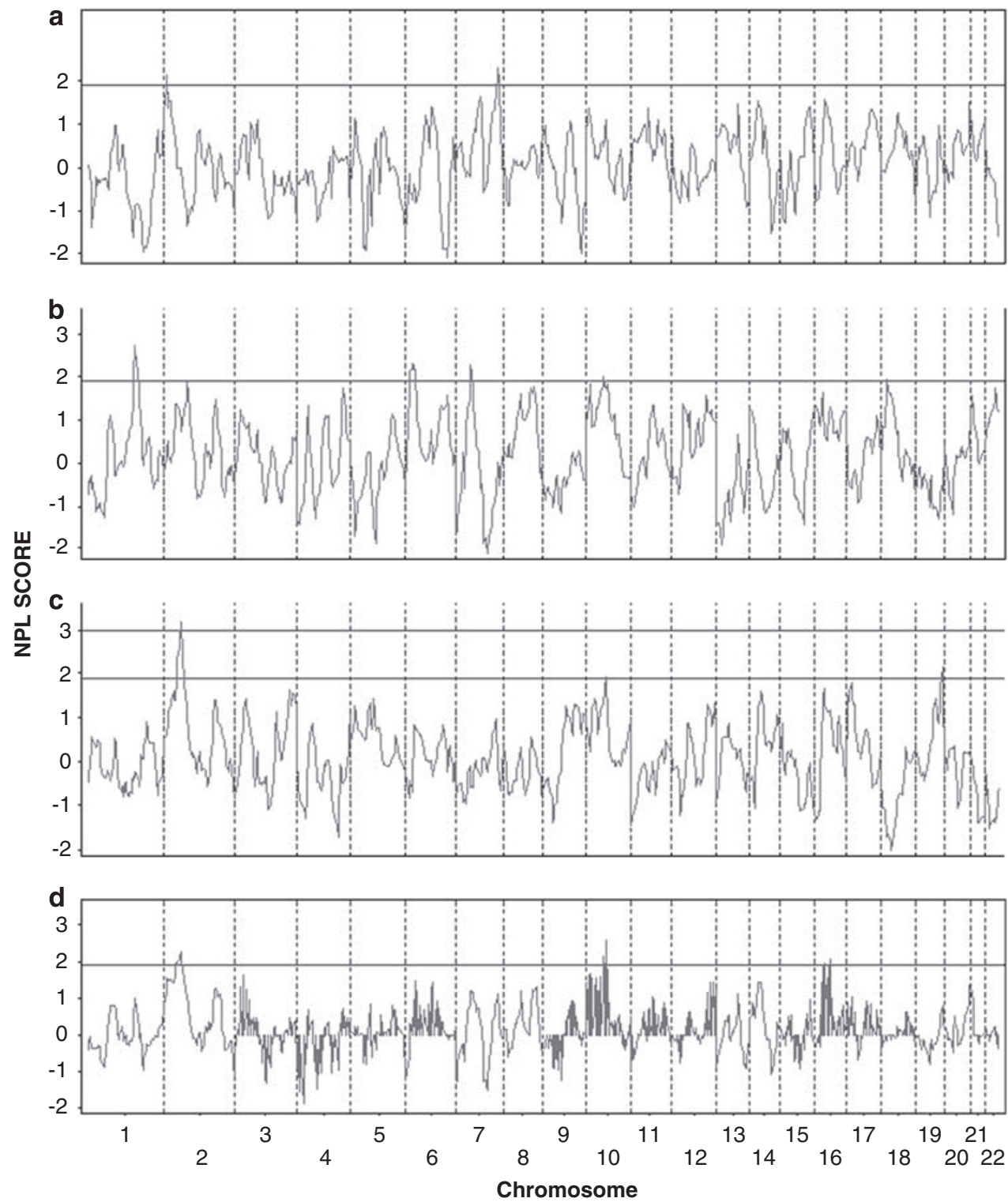

Figure 2 Genome-wide non-parametric LOD score results for 43 families with left ventricular outflow tract (LVOT) malformations in the initial 10 cM scan, by chromosome and position. NPL score (NPLS) results for (a) AVS families, (b) CoA families, (c) HLHS families, and (d) the combined LVOT malformation phenotype. The line indicates threshold for suggestive NPLS (1.9) and significant NPLS (3.0).

$65-80 \mathrm{cM}$ with overlaps of CoA and HLHS peaks. A clear increase in the combined NPLS can be observed with the co-occurrence of AVS, CoA, and HLHS peaks on chromosome 16 from position 35 to $45 \mathrm{cM}$.

\section{Sequencing}

The SMYD1 gene is situated at the edge of the peak on chromosome 2. All 10 exons and the exon/intron boundaries were sequenced in at least one affected individual from each family. No segregating mutations were identified.

\section{Discussion}

This is the first study to perform linkage analysis on families with multiple LVOT malformations considered as a combined LVOT malformation phenotype. Three novel regions with suggestive linkage were identified on chromosomes 2p23.2, 10q21.2, and 16p12.2. All individual malformations also show suggestive linkage, with a significant result on $2 \mathrm{p} 23.2$ for the HLHS families. There is an overlap of some linkage peaks for the individual malformations, suggesting that the locus may contribute a general susceptibility to develop an LVOT malformation, rather than conferring risk for a specific malformation. 
Table 2 NPL and LOD scores for linkage analysis of 43 families with left ventricular outflow tract malformations

\begin{tabular}{|c|c|c|c|c|c|c|}
\hline Marker & Physical position (bp) & Genetic position (cM) & $\angle O D$ & alpha & $H L O D$ & $N P L S$ \\
\hline \multirow[t]{2}{*}{ D2S168 } & 11362901 & 29.14 & -28.0859 & 0.203 & 0.3557 & 1.4770 \\
\hline & & & -3.5028 & 0.409 & 1.2637 & 1.3787 \\
\hline \multirow[t]{2}{*}{ D2S305 } & 19281858 & 42.58 & -15.8609 & 0.309 & 1.4151 & 1.4137 \\
\hline & & & -2.7541 & 0.415 & 1.7789 & 1.5540 \\
\hline \multirow[t]{2}{*}{ D2S165 } & 28456785 & 52.00 & -7.1025 & 0.492 & 2.2007 & 1.9339 \\
\hline & & & -3.3247 & 0.475 & 1.8423 & 1.9227 \\
\hline \multirow[t]{2}{*}{ D2S367 } & 34294651 & 60.55 & -25.0467 & 0.328 & 1.1383 & 1.9927 \\
\hline & & & -8.9166 & 0.353 & 1.2083 & 2.0973 \\
\hline \multirow{2}{*}{ D2S2259 } & 42850050 & 70.36 & -19.2458 & 0.347 & 1.2153 & 2.2583 \\
\hline & & & -12.6881 & 0.342 & 1.1562 & 2.1626 \\
\hline \multirow[t]{2}{*}{ D2S391 } & 46264871 & 75.93 & -20.5398 & 0.327 & 1.0792 & 2.0870 \\
\hline & & & -8.4940 & 0.302 & 0.7888 & 1.4996 \\
\hline D10S208 & 31720082 & 59.63 & $\begin{array}{r}-13.6236 \\
-60721\end{array}$ & $\begin{array}{l}0.0990 \\
03330\end{array}$ & $\begin{array}{l}0.0424 \\
0.4980\end{array}$ & $\begin{array}{l}1.5883 \\
1.9308\end{array}$ \\
\hline \multirow[t]{2}{*}{ D10S196 } & 51812273 & 69.37 & -17.3778 & 0.2400 & 0.2607 & 2.1327 \\
\hline & & & -4.7211 & 0.3900 & 0.7525 & 2.2819 \\
\hline \multirow[t]{2}{*}{ D10S1652 } & 64077500 & 80.04 & -19.2786 & 0.3440 & 0.8879 & 2.5707 \\
\hline & & & -9.2428 & 0.2970 & 0.5244 & 2.1479 \\
\hline \multirow[t]{2}{*}{ D10S537 } & 72065333 & 89.34 & -31.5072 & 0.1130 & 0.0730 & 1.7875 \\
\hline & & & -10.1712 & 0.1810 & 0.2735 & 1.0040 \\
\hline \multirow[t]{2}{*}{ D16S3075 } & 12116698 & 29.42 & -30.7294 & 0.0670 & 0.0335 & 0.5389 \\
\hline & & & -9.1089 & 0.3680 & 0.9469 & 1.1543 \\
\hline \multirow[t]{2}{*}{ D16S3103 } & 17380963 & 37.82 & -17.7972 & 0.4580 & 1.8967 & 1.8979 \\
\hline & & & -4.3886 & 0.5210 & 2.0960 & 1.9359 \\
\hline \multirow[t]{2}{*}{ D16S3046 } & 20793898 & 43.87 & -14.0909 & 0.4770 & 1.7185 & 1.9631 \\
\hline & & & -2.5359 & 0.5600 & 2.2517 & 1.8773 \\
\hline \multirow[t]{2}{*}{ D16S3068 } & 25468101 & 51.16 & -21.4954 & 0.2900 & 0.6198 & 1.5409 \\
\hline & & & -6.6037 & 0.4070 & 1.1164 & 1.6606 \\
\hline \multirow[t]{2}{*}{ D16S3136 } & 49263733 & 62.37 & -11.2392 & 0.3820 & 1.2381 & 1.8806 \\
\hline & & & -8.8130 & 0.4070 & 1.4464 & 1.9403 \\
\hline \multirow[t]{2}{*}{ D16S415 } & 52228161 & 67.57 & -17.8935 & 0.4010 & 1.4825 & 2.0329 \\
\hline & & & -7.6875 & 0.3270 & 0.6573 & 1.2151 \\
\hline
\end{tabular}

Alpha, proportion of families contributing to linkage signal in HLOD score; bp, base pairs, based on NCBI reference genome sequence, build 36; cM, genetic recombination distance in centiMorgans; LOD, lod score assuming homogeneity (all families contributing to locus).

The lack of a single strong linkage signal reveals the locus heterogeneity and complex inheritance of the LVOT malformations. Not more than half of the families segregated at any one locus (see $\alpha$ values, Table 2). In addition, several families contributed to more than one peak, similar to the earlier observations for BAV. ${ }^{24}$ This result supports our previous multiplex relative risk analysis, which indicated that disease susceptibility may require one to six loci. ${ }^{17}$ Although this study had adequate power to identify single locus inheritance, we did not have the power to detect more complex inheritance patterns.

Earlier studies on $\mathrm{BAV}^{24}$ have detected linkage to chromosomes 18q21, 5q21, and 13q34, and BAV with calcific AVS presenting in later ages has been attributed to NOTCH1 on 9q34 (Garg et al $\left.{ }^{9}\right)$. The highest LOD score observed in this study for any of these loci occurred in one family (Pedigree 80) that had a LOD score of 1.20 at marker D18S61 (chromosome 18q22.2). This family was ascertained by a proband with congenital AVS. A paternal first cousin had CoA. The proband's sibling and father were subsequently found to have asymptomatic BAV. Thus, we were unable to replicate any of the earlier linkage peaks.

The strength of this study is the careful phenotyping performed by echocardiography. We were able to include many additional families solely on the basis of finding asymptomatic BAV among relatives of a proband, which would otherwise have been considered an isolated case in the family. Screening as many relatives as possible also allowed proper labeling of affected and unaffected individuals. Although it is possible to argue that inclusion of milder malformations would increase heterogeneity, multiple studies have observed the highly variable expressivity of the LVOT malformations. ${ }^{15-17,25}$ Additionally, in an earlier linkage analysis of $\mathrm{BAV}$, inclusion of associated CVMs resulted in a gain of linkage signal. ${ }^{24}$ Thus, not including these milder malformations would have resulted in a loss of power.

A potential drawback of our study is the phenotypic heterogeneity. Several lines of evidence point to a common etiology of AVS, BAV, CoA, and HLHS, supporting the 

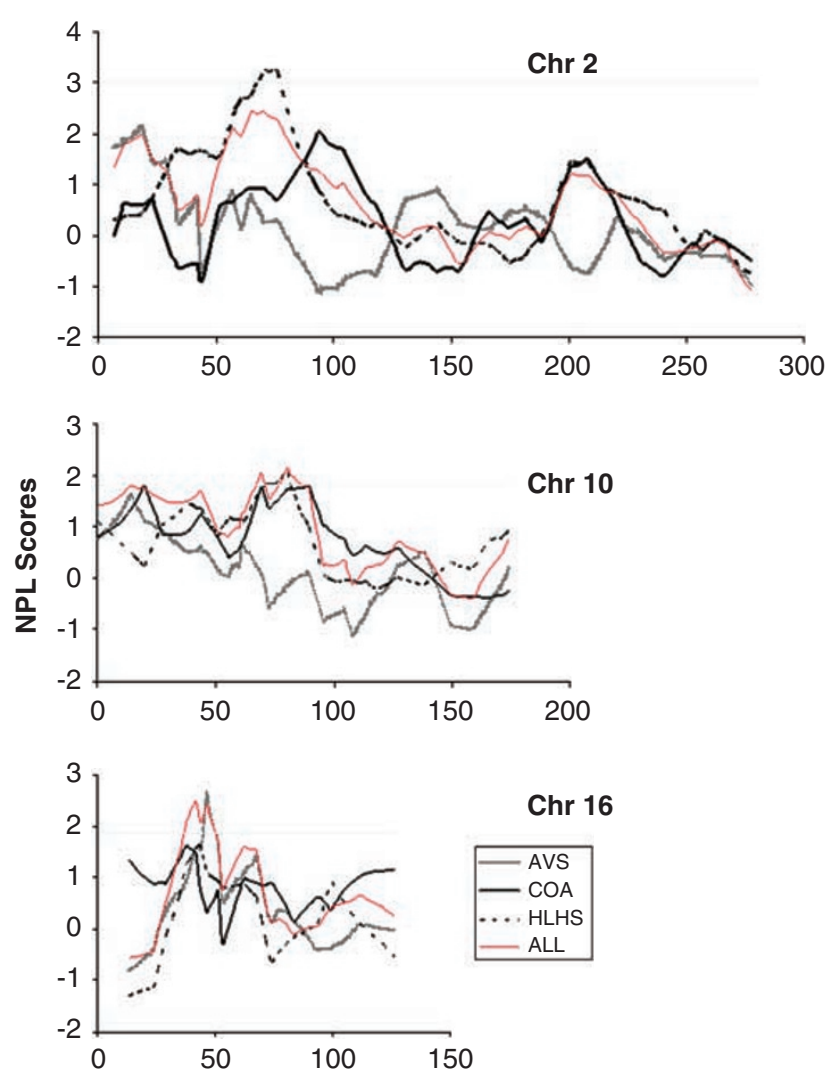

Figure 3 Linkage result NPLS for chromosomes 2, 10, and 16 with additional markers at $5 \mathrm{cM}$ intervals, by phenotype - ALL (combined LVOT), AVS, CoA, and HLHS. The line indicates threshold for suggestive NPLS (1.9) and significant NPLS (3.0).

choice to combine these families for analysis. Many epidemiology and family studies note the co-occurrence of different combinations of AVS, BAV, CoA, and HLHS within families. ${ }^{9,15-17,24-26}$ To ensure that we were not losing information by combining the phenotypes, we performed linkage analysis on each of the malformations individually. Contribution of each individual malformation to the highest peaks for the combined LVOT malformation varied from location to location. Greatest concordance of the peaks was observed for chromosome 16. Peaks for each malformation tended to cluster in the same location, although the peaks were diffuse and not always centered at the same point. Whether this is a function of the NPL analysis itself (which cannot derive precise locations, leading to broad linkage peaks) or separate, but closely placed, loci underlying each malformation is not known. Collection of more families with finer spaced genotyping may help answer this question.

We searched for possible candidate genes under the linkage peaks, based on knowledge learned from animal models. These models have shown that interacting molecular pathways are necessary for normal formation of the ventricles, their outflow tracts, semilunar valves, and respective great arteries. ${ }^{27,28}$ Mouse knock-out models of SMYD1, ${ }^{29}$ Nrg1 $^{30}{ }^{30}$ ErbB2,${ }^{31}$ ErbB4 ${ }^{32}$ and Notch $1^{33-35}$ exhibit primary ventricular hypoplasia or reduced wall thickness. The transcription factor NFATC is implicated in semilunar valve formation, ${ }^{36,37}$ whereas mice heterozygous for eNos mutation show BAV. ${ }^{38}$ Mutations in endothelin 1 (Edn1), ${ }^{39}$ endothelin-A receptor (Ednra) and ECE1, ${ }^{40,41}$ and gridlock/ HEY2/HRT2 ${ }^{42-44}$ (among others) lead to aortic arch malformations.

In addition to intrinsic pattern formation, cardiomyocyte, endocardium, valve, and great vessel morphogenesis requires blood-flow-directed remodeling. Primary malformations that affect blood flow into either ventricle may result in secondary hypoplasia, as seen in chick, ${ }^{45}$ fetal lamb, ${ }^{46}$ and zebrafish ${ }^{47}$ models in which restriction of LV inflow produces a phenotype similar to HLHS, and restoration of blood flow rescues the phenotype. ${ }^{48}$ Corroborative human evidence has been found by prenatal ultrasounds in fetuses affected with HLHS, which show worsening of LV hypoplasia as gestation progresses. ${ }^{49,50}$ This secondary effect, along with other differences between humans and mouse, may explain why there are no animal models that precisely mimic LVOT malformations.

One gene, SMYD1 on chromosome 2, was a particularly compelling candidate as the mouse knock-out model shows ventricular hypoplasia. ${ }^{29}$ We were not able to identify segregating mutations in this gene, however. Given the broad linkage peaks, investigation of other candidates will require a narrowing of the list through fine mapping before proceeding further.

We have recently identified NOTCH1 mutations that alter Notch signaling in human subjects with sporadic AVS, CoA, and HLHS. Our lack of linkage to the NOTCH1 locus on 9q34 highlights the complex genetic nature of the LVOT malformations, and the strengths and weaknesses of different techniques used to identify the precise gene defects. Linkage analysis is a powerful approach to identify the involvement of a highly penetrant gene, particularly if applied to a single large family. It performs poorly if there are multiple common but weakly penetrant susceptibility loci, which are better identified in genome-wide association studies. In turn, multiple rare variants in one gene (acting in concert with other loci) will not be found in association studies. These will only be identified by resequencing of candidate genes, the technique we used to identify rare NOTCH1 susceptibility variants in AVS, CoA, and HLHS subjects. Thus, it is likely that all these techniques (linkage analysis of large families, association studies in isolated affected individuals, and resequencing) will be required to uncover the genetic etiology of LVOT malformations.

In summary, we report suggestive evidence for linkage to chromosomes 2p23, 10q21, and 16p12 for the combined LVOT malformation phenotype and for the individual 
phenotypes of AVS, CoA, and HLHS, with a significant linkage signal for HLHS on 2p23. Additional suggestive peaks unique to AVS (16p12, 7q36), CoA (1q24, 6p23, $7 \mathrm{p} 14)$, and HLHS (19q13) were also found. This provides the initial phase for gene discovery for these malformations, and offers support to the concept of common etiologies and perhaps a final common pathway influencing all these malformations. Further collection of pedigrees and the addition of fine mapping are currently in progress to confirm our initial results and narrow the regions for candidate gene screening.

\section{Acknowledgements}

Funding support: NIH HL70823 and HD39056, and the Research Institute at Nationwide Children's Hospital to KLM, and the NIH HD43372 to JWB. The authors declare no conflicts of interest.

\section{References}

1 Hoffman JI, Kaplan S: The incidence of congenital heart disease. J Am Coll Cardiol 2002; 39: 1890-1900.

2 McBride KL, Marengo L, Canfield M, Langlois P, Fixler D, Belmont JW: Epidemiology of noncomplex left ventricular outflow tract obstruction malformations (aortic valve stenosis, coarctation of the aorta, hypoplastic left heart syndrome) in Texas, 1999-2001. Birth Defects Res A Clin Mol Teratol 2005; 73: 555-561.

3 Pradat P, Francannet C, Harris JA, Robert E: The epidemiology of cardiovascular defects, part I: a study based on data from three large registries of congenital malformations. Pediatr Cardiol 2003; 24: $195-221$.

4 Alsoufi B, Bennetts J, Verma S, Caldarone CA: New developments in the treatment of hypoplastic left heart syndrome. Pediatrics 2007; 119: 109-117.

5 Jenkins KJ, Correa A, Feinstein JA et al: Noninherited risk factors and congenital cardiovascular defects: current knowledge: a scientific statement from the American Heart Association Council on Cardiovascular Disease in the Young: endorsed by the American Academy of Pediatrics. Circulation 2007; 115: $2995-3014$.

6 Gotzsche CO, Krag-Olsen B, Nielsen J, Sorensen KE, Kristensen BO: Prevalence of cardiovascular malformations and association with karyotypes in Turner's syndrome. Arch Dis Child 1994; 71: $433-436$.

7 Grossfeld PD, Mattina T, Lai Z et al: The 11q terminal deletion disorder: a prospective study of 110 cases. Am J Med Genet 2004; 129A: $51-61$.

8 Ferencz C, Neill CA, Boughman JA, Rubin JD, Brenner JI, Perry LW: Congenital cardiovascular malformations associated with chromosome abnormalities: an epidemiologic study. I Pediatr 1989; 114: 79-86.

9 Garg V, Muth AN, Ransom JF et al: Mutations in NOTCH1 cause aortic valve disease. Nature 2005; 437: 270-274.

10 McKellar SH, Tester DJ, Yagubyan M, Majumdar R, Ackerman MJ, Sundt III TM: Novel NOTCH1 mutations in patients with bicuspid aortic valve disease and thoracic aortic aneurysms. J Thorac Cardiovasc Surg 2007; 134: 290-296.

11 Mohamed SA, Aherrahrou Z, Liptau H et al: Novel missense mutations (p.T596M and p.P1797H) in NOTCH1 in patients with bicuspid aortic valve. Biochem Biophys Res Commun 2006; 345: $1460-1465$.

12 McBride KL, Riley MF, Zender GA et al: NOTCH1 mutations in individuals with left ventricular outflow tract malformations reduce ligand-induced signaling. Hum Mol Genet 2008; 17: $2886-2893$.
13 Elliott DA, Kirk EP, Yeoh T et al: Cardiac homeobox gene NKX2-5 mutations and congenital heart disease: associations with atrial septal defect and hypoplastic left heart syndrome. J Am Coll Cardiol 2003; 41: 2072-2076.

14 McElhinney DB, Geiger E, Blinder J, Benson DW, Goldmuntz E: NKX2.5 mutations in patients with congenital heart disease. J Am Coll Cardiol 2003; 42: 1650-1655.

15 Lewin MB, McBride KL, Pignatelli $\mathrm{R}$ et al: Echocardiographic evaluation of asymptomatic parental and sibling cardiovascular anomalies associated with congenital left ventricular outflow tract lesions. Pediatrics 2004; 114: 691-696.

16 Loffredo CA, Chokkalingam A, Sill AM et al: Prevalence of congenital cardiovascular malformations among relatives of infants with hypoplastic left heart, coarctation of the aorta, and d-transposition of the great arteries. Am J Med Genet 2004; 124A: 225-230.

17 McBride KL, Pignatelli R, Lewin M et al: Inheritance analysis of congenital left ventricular outflow tract obstruction malformations: segregation, multiplex relative risk, and heritability. Am J Med Genet A 2005; 134: 180-186.

$18 \mathrm{O}^{\prime}$ Connell JR, Weeks DE: PedCheck: a program for identification of genotype incompatibilities in linkage analysis. Am J Hum Genet 1998; 63: 259-266.

19 Abecasis GR, Cherny SS, Cookson WO, Cardon LR: Merlin-rapid analysis of dense genetic maps using sparse gene flow trees. Nat Genet 2002; 30: 97-101.

20 Gudbjartsson DF, Jonasson K, Frigge ML, Kong A: Allegro, a new computer program for multipoint linkage analysis. Nat Genet 2000; 25: 12-13.

21 Terwilliger JD, Speer M, Ott J: Chromosome-based method for rapid computer simulation in human genetic linkage analysis. Genet Epidemiol 1993; 10: 217-224.

22 Ott J: Computer-simulation methods in human linkage analysis. Proc Natl Acad Sci USA 1989; 86: 4175-4178.

23 Weeks D, Ott J, Lathrop G: SLINK: a general simulation program for linkage analysis. Am J Hum Genet 1990; 47: A204.

24 Martin LJ, Ramachandran V, Cripe LH et al: Evidence in favor of linkage to human chromosomal regions $18 \mathrm{q}, 5 \mathrm{q}$ and $13 \mathrm{q}$ for bicuspid aortic valve and associated cardiovascular malformations. Hum Genet 2007; 121: 275-284.

25 Wessels MW, Berger RM, Frohn-Mulder IM et al: Autosomal dominant inheritance of left ventricular outflow tract obstruction. Am J Med Genet A 2005; 134: 171-179.

26 Ferencz C, Loffredo CA, Corea-Vilasenor A, Wilson PD: Left sided obstructive lesions;in Anderson R (ed):: Genetic and Environmental Risk Factors of Major Cardiovascular Malformations. Armonk, NY: Futura Publishing Co. Inc.,, 1997, Vol 5, pp 165-225.

27 Brand T: Heart development: molecular insights into cardiac specification and early morphogenesis. Dev Biol 2003; 258: 1-19.

28 Bruneau BG: Transcriptional regulation of vertebrate cardiac morphogenesis. Circ Res 2002; 90: 509-519.

29 Gottlieb PD, Pierce SA, Sims RJ et al: Bop encodes a musclerestricted protein containing MYND and SET domains and is essential for cardiac differentiation and morphogenesis. Nat Genet 2002; 31: 25-32.

30 Meyer D, Birchmeier C: Multiple essential functions of neuregulin in development. Nature 1995; 378: 386-390.

31 Lee KF, Simon H, Chen H, Bates B, Hung MC, Hauser C: Requirement for neuregulin receptor erbB2 in neural and cardiac development. Nature 1995; 378: 394-398.

32 Gassmann M, Casagranda F, Orioli D et al: Aberrant neural and cardiac development in mice lacking the ErbB4 neuregulin receptor. Nature 1995; 378: 390-394.

33 Grego-Bessa J, Luna-Zurita L, del Monte G et al: Notch signaling is essential for ventricular chamber development. Dev Cell 2007; 12: $415-429$.

34 Krebs LT, Xue Y, Norton CR et al: Notch signaling is essential for vascular morphogenesis in mice. Genes Dev 2000; 14: 1343-1352.

35 Timmerman LA, Grego-Bessa J, Raya A et al: Notch promotes epithelial-mesenchymal transition during cardiac 
development and oncogenic transformation. Genes Dev 2004; 18 : 99-115.

36 de la Pompa JL, Timmerman LA, Takimoto $\mathrm{H}$ et al: Role of the NF-ATc transcription factor in morphogenesis of cardiac valves and septum. Nature 1998; 392: 182-186.

37 Ranger AM, Grusby MJ, Hodge MR et al: The transcription factor NF-ATc is essential for cardiac valve formation. Nature 1998; 392: $186-190$.

38 Lee TC, Zhao YD, Courtman DW, Stewart DJ: Abnormal aortic valve development in mice lacking endothelial nitric oxide synthase. Circulation 2000; 101: 2345-2348.

39 Kurihara $\mathrm{Y}$, Kurihara $\mathrm{H}$, Oda $\mathrm{H}$ et al: Aortic arch malformations and ventricular septal defect in mice deficient in endothelin-1. I Clin Invest 1995; 96: 293-300.

40 Yanagisawa H, Hammer RE, Richardson JA et al: Disruption of ECE-1 and ECE-2 reveals a role for endothelin-converting enzyme-2 in murine cardiac development. J Clin Invest 2000; 105: 1373-1382.

41 Yanagisawa H, Hammer RE, Richardson JA, Williams SC, Clouthier DE, Yanagisawa M: Role of endothelin-1/endothelin-A receptor-mediated signaling pathway in the aortic arch patterning in mice. J Clin Invest 1998; 102: 22-33.

42 Gessler M, Knobeloch KP, Helisch A et al: Mouse gridlock: no aortic coarctation or deficiency, but fatal cardiac defects in Hey2-/- mice. Curr Biol 2002; 12: 1601-1604.
43 Weinstein BM, Stemple DL, Driever W, Fishman MC: Gridlock, a localized heritable vascular patterning defect in the zebrafish. Nat Med 1995; 1: 1143-1147.

44 Zhong TP, Rosenberg M, Mohideen MA, Weinstein B, Fishman MC: Gridlock, an HLH gene required for assembly of the aorta in zebrafish. Science 2000; 287: 1820-1824.

45 Sedmera D, Pexieder T, Rychterova V, Hu N, Clark EB: Remodeling of chick embryonic ventricular myoarchitecture under experimentally changed loading conditions. Anat Rec 1999; 254: $238-252$.

46 Samson F, Bonnet N, Heimburger $\mathrm{M}$ et al: Left ventricular alterations in a model of fetal left ventricular overload. Pediatr Res 2000; 48: 43-49.

47 Hove JR, Koster RW, Forouhar AS, Acevedo-Bolton G, Fraser SE, Gharib M: Intracardiac fluid forces are an essential epigenetic factor for embryonic cardiogenesis. Nature 2003; 421: 172-177.

48 deAlmeida A, McQuinn T, Sedmera D: Increased ventricular preload is compensated by myocyte proliferation in normal and hypoplastic fetal chick left ventricle. Circ Res 2007; 100: 1363-1370.

49 Danford DA, Cronican P: Hypoplastic left heart syndrome: progression of left ventricular dilation and dysfunction to left ventricular hypoplasia in utero. Am Heart J 1992; 123: 1712-1713.

50 Hornberger LK, Need L, Benacerraf BR: Development of significant left and right ventricular hypoplasia in the second and third trimester fetus. J Ultrasound Med 1996; 15: 655-659.

Supplementary Information accompanies the paper on European Journal of Human Genetics website (http://www.nature.com/ejhg) 